\title{
Collagen XIV Is an Intrinsic Regulator of Corneal Stromal Structure and Function
}

Mei Sun, ${ }^{*}$ Nabeel Zafrullah, ${ }^{*}$ Sheila Adams, ${ }^{*}$ Floriane Devaux, ${ }^{\dagger}$ Marcel Y. Avila, ${ }^{\ddagger}$ Noel Ziebarth, ${ }^{\dagger \S}$ Curtis E. Margo, ${ }^{*}$ Manuel Koch," and Edgar M. Espana*\|

From the Cornea and External Disease, * Department of Ophthalmology, and the Department of Molecular Pharmacology and Physiology," University of South Florida, Morsani College of Medicine, Tampa, Florida; the Biomedical Atomic Force Microscopy Laboratory, ${ }^{\dagger}$ Department of Biomedical Engineering, College of Engineering, and the Department of Physiology and Biophysics, ${ }^{\complement}$ Miller School of Medicine, University of Miami, Miami, Florida; the Department of Ophthalmology, ${ }^{\ddagger}$ Universidad Nacional de Colombia, Bogota, Colombia; and the Institute for Dental Research and Oral Musculoskeletal Biology, ${ }^{\llbracket}$ Center for Biochemistry, University of Cologne, Cologne, Germany

Accepted for publication

August 16, 2021.

Address correspondence to Edgar M. Espana, M.D., Department of Ophthalmology, University of South Florida, Morsani College of Medicine, 13330 USF Laurel Dr., 4th floor, MDC11, Tampa, FL 33612. E-mail: eespana@usf edu.

\begin{abstract}
Collagen XIV is poorly characterized in the body, and the current knowledge of its function in the cornea is limited. The aim of the current study was to elucidate the role(s) of collagen XIV in regulating corneal stromal structure and function. Analysis of collagen XIV expression, temporal and spatial, was performed at different postnatal days (Ps) in wild-type C57BL/6 mouse corneal stromas and after injury. Conventional collagen XIV null mice were used to inquire the roles that collagen XIV plays in fibrillogenesis, fibril packing, and tissue mechanics. Fibril assembly and packing as well as stromal organization were evaluated using transmission electron microscopy and second harmonic generation microscopy. Atomic force microscopy was used to assess stromal stiffness. Col14a1 mRNA expression was present at P4 to P10 and decreased at P30. No immunoreactivity was noted at P150. Abnormal collagen fibril assembly with a shift toward larger-diameter fibrils and increased interfibrillar spacing in the absence of collagen XIV was found. Second harmonic generation microscopy showed impaired fibrillogenesis in the collagen XIV null stroma. Mechanical testing suggested that collagen XIV confers stiffness to stromal tissue. Expression of collagen XIV is up-regulated following injury. This study indicates that collagen XIV plays a regulatory role in corneal development and in the function of the adult cornea. The expression of collagen XIV is recapitulated during wound healing. (Am J Pathol 2021, 191: 2184-2194; https://doi.org/10.1016/j.ajpath.2021.08.016)
\end{abstract}

Fibril-associated collagens with interrupted triple helices (FACITs), located in the interfibrillar spaces, regulate the supramolecular organization of fibrils and are critical for tissue integrity, biomechanical stability, and function. ${ }^{1}$ Collagen XIV is a homotrimer that contains two collagen triple-helical domains (COL1 and COL2) and three noncollagenous domains (NC1, NC2, and NC3). ${ }^{1-4}$ The short triple-helical domains interact with and adhere to the surface of fibrillar collagens. ${ }^{3}$ Although some domains of collagen XIV interact with collagen I, the COL2 domain sticks out of the fibril and projects the large NC3 domain, a large amino terminal globular domain, away from the fibril into the interfibrillar space. ${ }^{5}$

Collagen XIV expression in avians, ${ }^{2,5,6}$ bovine, ${ }^{7,8}$ mouse, ${ }^{9-11}$ and human tissues ${ }^{12,13}$ is prevalent in skin, tendon, cornea, cartilage, and myocardium. The role that collagen XIV plays in these different tissues is poorly understood, but it is thought to contribute mechanical strength. Collagen XIV is expressed in areas that incur high mechanical stress. Such tissues include tendonous attachments to bones, 9,14 perifollicular hair shafts, where support of the follicle is critical, $^{13}$ and peripheral nerve tissues, like epineurium and perineurium. ${ }^{5}$

The role of collagen XIV in the cornea is not understood. Collagen XIV has been implicated in the regulation of corneal stromal compaction in the chick. ${ }^{6,15}$ Our laboratory is interested in defining the roles of FACITs, collagens XII

Supported by NIH/National Eye Institute grant EY029395 (E.M.E.).

Disclosures: None declared. 
and XIV, in corneal structure and function. ${ }^{11,16}$ The purpose of this study was to establish the functional role(s) of collagen XIV in the development and function of the corneal stroma.

\section{Materials and Methods}

\section{Animals}

Wild-type C57BL/6 (WT) and gene-targeted mice null in collagen XIV on a C57BL/6 background $\left(\mathrm{Coll}_{14 \mathrm{al}^{-1-}}\right)$ were used. ${ }^{9}$ The Coll $_{4 a 1^{-1}}$ cornea did not express collagen $\mathrm{XIV}$, as previously demonstrated. ${ }^{11}$

\section{RNA Isolation and Quantification of mRNA}

Whole corneas were dissected from WT mice at days of age 4, 10, 30, 90, and 150 and in adult mice, 60 days old; and after 1 day and 1, 3, and 6 weeks of generating an injury, as previously described. ${ }^{17}$ The isolated corneas were incubated with Dispase II (Roche, Indianapolis, IN) to remove corneal epithelium. ${ }^{18}$ The corneal stroma was cut into small pieces, and total RNA was extracted using QIAzol Lysis Reagent (Qiagen, Hilden, Germany) and RNeasy MinElute Cleanup Kit (Qiagen). Reverse transcription and quantitative realtime PCR analysis were performed as previously described. ${ }^{16}$ The following primer sequences were used: Coll4al, 5'-ACCTGTGAGTGTCCCTGGTC-3' (forward) and $5^{\prime}$-AGGCCAGTCAGAGCATCACT- $3^{\prime}$ (reverse); and $A c t b, 5^{\prime}$-AGATGACCCAGATCATGTTTGAGA-3' (forward) and 5'-CACAGCCTGGATGGCTACGT-3' (reverse). Each sample was run in duplicate PCR. Three to five corneas of different mice at each age were used. Experiments were repeated three times.

\section{Quantitation of Protein Expression}

The stroma from WT and Coll4a1 ${ }^{-1}$ mice at different ages were cut into small pieces. Stromas from three to eight mice were pooled, and protein was extracted using radioimmunoprecipitation assay lysis buffer. Protein concentration was determined through a bicinchoninic acid assay (Thermo Scientific Pierce, Waltham, MA). Collagen XIV protein expression was analyzed using Simple Westerns (WES; ProteinSimple, San Jose, CA) simple Western system, strictly following the manufacturer's instructions. A 12- to 230-kDa separation module, an anti-rabbit detection module, and a total protein detection module were applied. Briefly, samples diluted with $0.1 \times$ sample buffer (ProteinSimple) at concentration of $0.125 \mu \mathrm{g} / \mathrm{mL}$ were loaded and hybridized with anti-collagen XIV antibody (1:500 dilution; clone KR47; Dr. Manuel Koch, University of Cologne, Cologne, Germany). ${ }^{11}$ Total protein was used as a loading control. The error bars are from two sets of WES experiment, which use two sets of protein samples. Each set
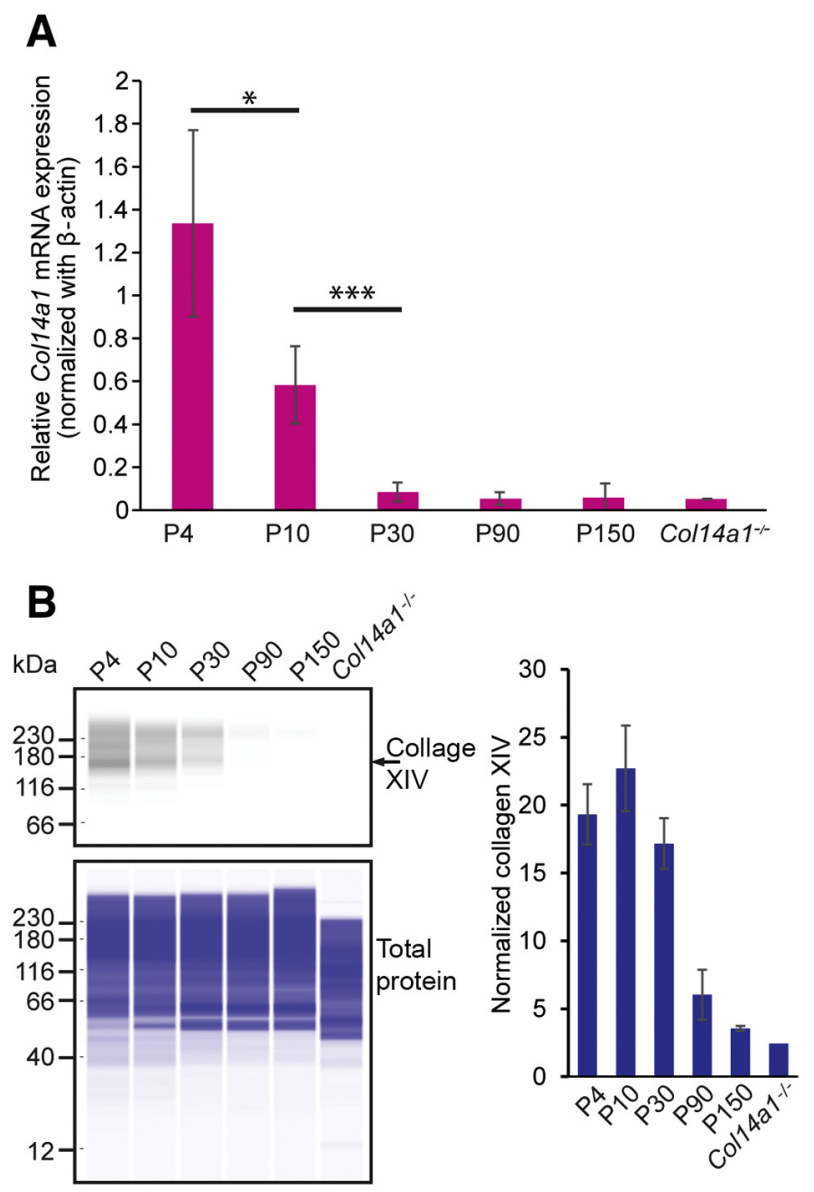

Figure 1 Col14a1 expression in the cornea decreases with maturation, and collagen XIV expression weakens in early adulthood. A: Col14a1 mRNA expression (pink) in the mouse cornea decreases after postnatal day (P) 4, shown by normalized Col14a1 mRNA expression in real-time PCR analysis. B: Protein expression of collagen XIV was decreased at P30 and mostly absent at P150. Left panel: The representative WES images of collagen XIV expression and total protein. Right panel: The quantitation of the collagen XIV normalized with total protein. The error bars represent the SD from two independent experiments. Three to five corneas of different mice at each age were used. Experiments were repeated three times. ${ }^{*} P<0.05,{ }^{* *} P<$ 0.001 .

of protein contains cornea lysate from 3 to 8 mice (6 to 16 corneas) in each age.

\section{Immunofluorescence Microscopy}

Fresh eyes were harvested from WT mice at different ages, 4 to 150 days old, and then embedded in OCT medium and frozen with isopentane (Sigma Aldrich, St. Louis, MO) on dry ice. Corneal sections ( $5 \mu \mathrm{m}$ thick) were fixed with cold acetone, blocked using 10\% donkey serum (Sigma Aldrich), and then incubated with rabbit anti-collagen XIV antibody (1:200 dilution; KR47) $)^{11}$ for 2 hours at room temperature. The secondary antibodies were Alexa Fluor 488 or 594 donkey anti-rabbit IgG (Invitrogen, Carlsbad, CA) used at 1:200. Vectashield mounting solution with DAPI was used as a cell nuclei marker (Vector Laboratories, Burlingame, $\mathrm{CA})$. Images were captured using a fluorescence microscope 

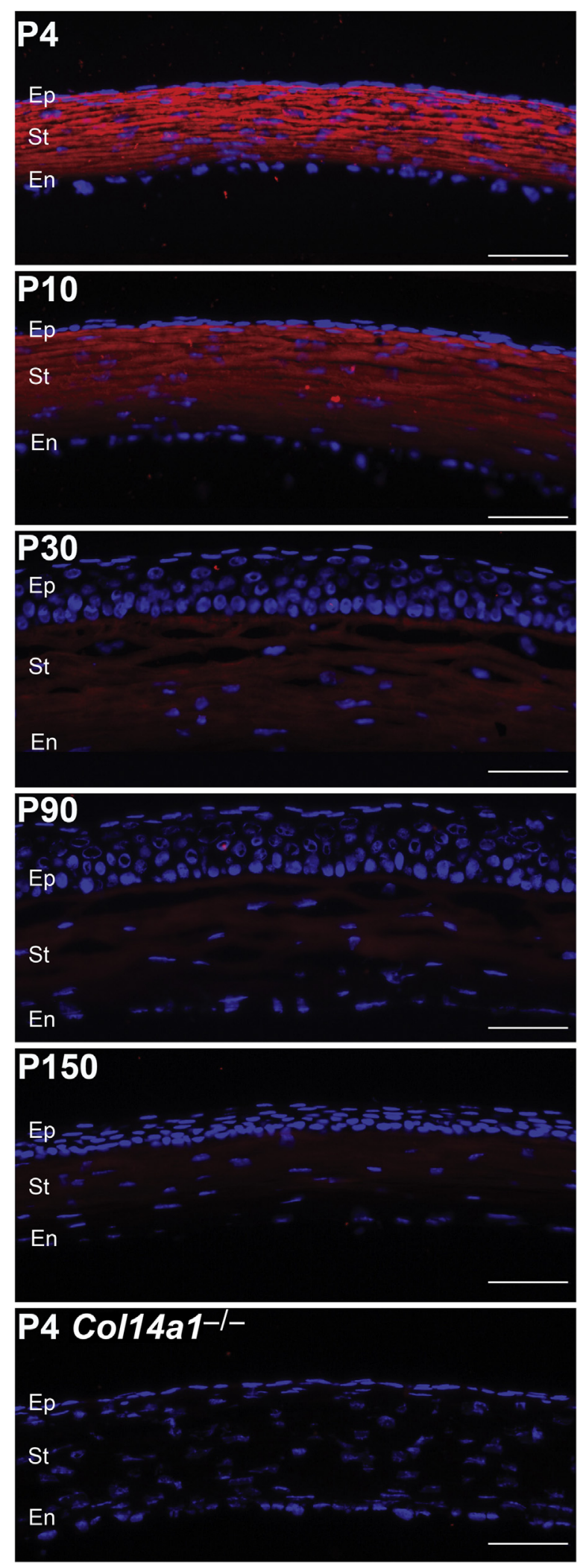

Figure 2 Collagen XIV localizes throughout the entire corneal stroma (St). Collagen XIV (red) localizes throughout the entire corneal stroma during development from postnatal day (P) 4 and P30. Immunoreactivity decreases at P30 and mostly localizes to the anterior stroma at P90 and P150. A P4 Col14a1 ${ }^{-/-}$cornea is used as the negative control. $n=3$. Scale bars $=50 \mu \mathrm{m}$. En, endothelium; Ep, epithelium.
(Leica, Wetzlar, Germany; DM5500B). Identical conditions and negative controls facilitated comparisons between samples. Staining was repeated using same conditions at least three times for consistency.

\section{SHG Microscopy of Corneal Stroma}

Enucleated eyes were immediately placed in Optisol media on a custom-made glass chamber and imaged (within 5 minutes) without any tissue manipulation or additional dissection. Corneal cross-sections were imaged using an Olympus MPE-RS microscope using a $25 \times(0.95$ numerical aperture) water-immersion objective (Olympus Corp., Tokyo, Japan). Two-photon second harmonic generation (SHG) signals were generated using a mode-locked titanium/sapphire laser at $960 \mathrm{~nm}$. The SHG forward-scattered signals passing through the corneal sections were collected using a 0.8 numerical aperture condenser lens with a narrow band-pass filter (465 to $485 \mathrm{~nm}$ ). Backward-scattered SHG signals were detected with a band-pass filter (460 to 500 $\mathrm{nm})$. All samples were scanned using a $2-\mu \mathrm{m} z$-axis step size from the back to the front of the section. Ten continuous slices were randomly selected from a stack of images obtained from the central corneal stroma and analyzed using ImageJ software version 2.10/1.53C (NIH, Bethesda, MD; https://imagej.nih.gov/ij). A mean pixel intensity value was obtained from each slice as an approximation of fibril density. Inferences in the differences in collagen XIV null stromas and control WT pixel intensity were tested for using $t$-test. Four corneas at different ages were used.

\section{Transmission Electron Microscopy}

Cornea samples from WT and Coll4a1 ${ }^{-1-}$ mice, 30 days old, were analyzed using transmission electron microscopy (TEM), as previously described. ${ }^{16}$ Briefly, at least three corneas per group were dissected and fixed in $4 \%$ paraformaldehyde, $2.5 \%$ glutaraldehyde, $0.1 \mathrm{~mol} / \mathrm{L}$ sodium cacodylate, $\mathrm{pH} 7.4$, with $8.0 \mathrm{mmol} / \mathrm{L} \mathrm{CaCl}$, post-fixed in $1 \%$ osmium tetroxide. The corneas were dehydrated in graded ethanol series, followed by propylene oxide. The tissue samples were infiltrated and embedded in a mixture of Embed 812, nadic methyl anhydride, dodecenyl succinic anhydride, and DMP-30 (Electron Microscopy Sciences, Hatfield, PA). Thin sections (approximately $80 \mathrm{~nm}$ ) were cut with a Leica ultramicrotome and post-stained with $2 \%$ aqueous uranyl acetate and $1 \%$ phosphotungstic acid, $\mathrm{pH}$ 3.2. The sections were examined at $80 \mathrm{kV}$ with a JEOL 1400 transmission electron microscope (Jeol, Tokyo, Japan) equipped with a Gatan Ultrascan US1000 2 K digital camera (Ametek, Berwin, PA).

\section{Fibril Diameter and Fibril Spacing Distribution}

Three corneas from three different WT and Col14al ${ }^{-1-}$ mice at postnatal day (P) 30 were analyzed. Six digital 

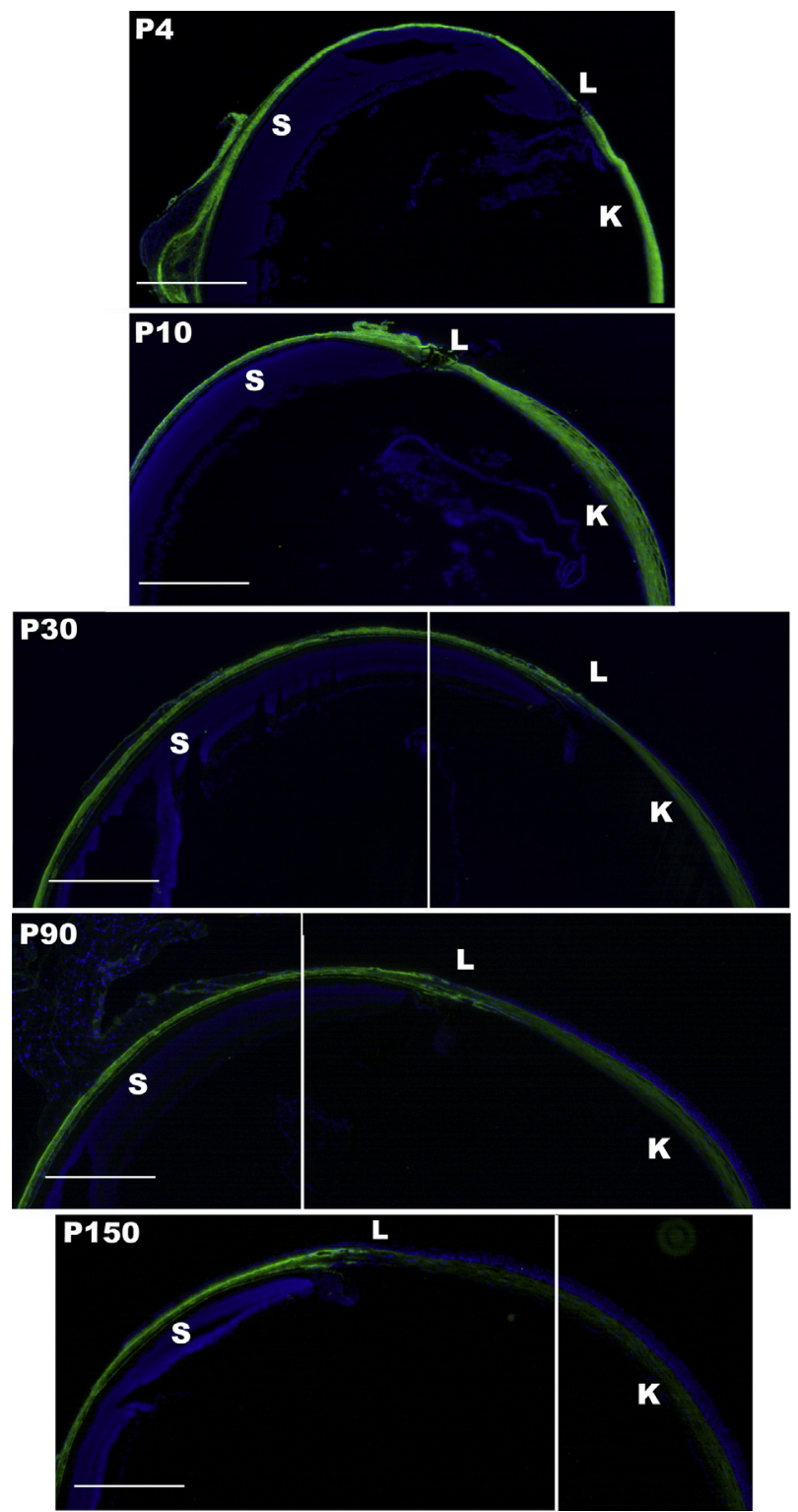

Figure 3 Composite images show collagen XIV expression persistence in the scleral stroma of adult eyes. Collagen XIV expression in wild-type C57BL/6 eye is persistent in the adult sclera (S), whereas corneal expression is rapidly decreasing. $n=3$. Scale bars $=450 \mu \mathrm{m}$. K, cornea; $\mathrm{L}$, limbal region; $\mathrm{P}$, postnatal day.

images per cornea were taken from nonoverlapping regions in the central stroma at a magnification of $\times 1,000,000$. The images were de-identified before fibril diameter and density were measured using Fiji's ImageJ software. A region of interest (ROI) of appropriate size was chosen within each image, where fibrils were perpendicular/cross-sectional to the viewing plane. Two to four ROIs that included a minimum of 80 fibrils were measured for each image. Equivalent radius calculates the radius that would contain an area equal to that of the object of interest in ImageJ software, which was chosen to represent the fibril diameters. Data analysis, histograms of the diameter distribution, and the Kolmogorov-Smirnov test were generated using Excel 2013
(Microsoft, Redmond, WA). Fibril density was obtained as the fibril number per unit area.

\section{Optical Coherence Tomography and Corneal Thickness Estimation}

Whole eyes from euthanized mice were enucleated, and measurements were immediately obtained. Each enucleated eye was placed in a custom-made holder and placed in the Spectral Dominium Cirrus HDT Optical Coherence Tomography (Zeiss, San Francisco, CA) device for corneal thickness measurements. ${ }^{11}$ Five measurements were obtained on the vertical plane and five measurements were obtained on the horizontal plane of the central cornea. Eight corneas from each phenotype were measured.

\section{Tissue Stiffness and Compression Resistance in Adult Corneas}

To analyze if collagen XIV influences corneal stiffness, atomic force microscopy measurements were performed on adult male when stromal maturation and compaction were believed to be completed, at 130 to 140 days of age. Measurements were obtained from WT and $\mathrm{Coll}_{4} \mathrm{al}^{-1-}$ corneas. Corneas were shipped overnight in Optisol solution for measurement using a laboratory-built atomic force microscopy measurement system specific for corneal compression measurements. On arrival in the laboratory, corneas were transferred to a $15 \%$ dextran solution to maintain hydration and isotonic conditions that would not affect stromal hydration during experiments. The measurement protocol has been described in detail in a recent publication. ${ }^{16}$ Briefly, elasticity was determined via indentation of corneas using a silicon nitride cantilever with a $2.5-\mu \mathrm{m}$ silica bead affixed to the apex $(\mathrm{k}=0.12 \mathrm{~N} / \mathrm{m}$; Novascan Technologies, Inc., Ames, IA). Measurements were repeated 10 times in at least three different locations around the cornea. Young's modulus of elasticity was calculated from the measurements by applying the Hertz model for a spherical indenter. Sixteen corneas were measured, eight of each phenotype.

\section{Stromal Injury}

Only left corneas were subjected to injury. All experiments conformed to the use of Laboratory Animals and Association for Research in Vision and Ophthalmology Statement for the Use of Animals in Ophthalmic and Vision Research and were approved by the Institutional Animal Care and Use Committee of the University of South Florida College of Medicine (Tampa, FL). P60 male WT mice were anesthetized by i.p. injection of ketamine $(100 \mathrm{mg} / \mathrm{kg})$ and xylazine $(10 \mathrm{mg} / \mathrm{kg})$. Once they were under general anesthesia, s.c. $1 \mathrm{mg} / \mathrm{kg}$ buprenorphine sustained-released analgesia was given. A total of $0.1 \mathrm{~mL}$ of $1 \%$ proparacaine hydrochloride and $1 \%$ atropine was placed on the ocular surface. Under microscope visualization, a partial thickness 

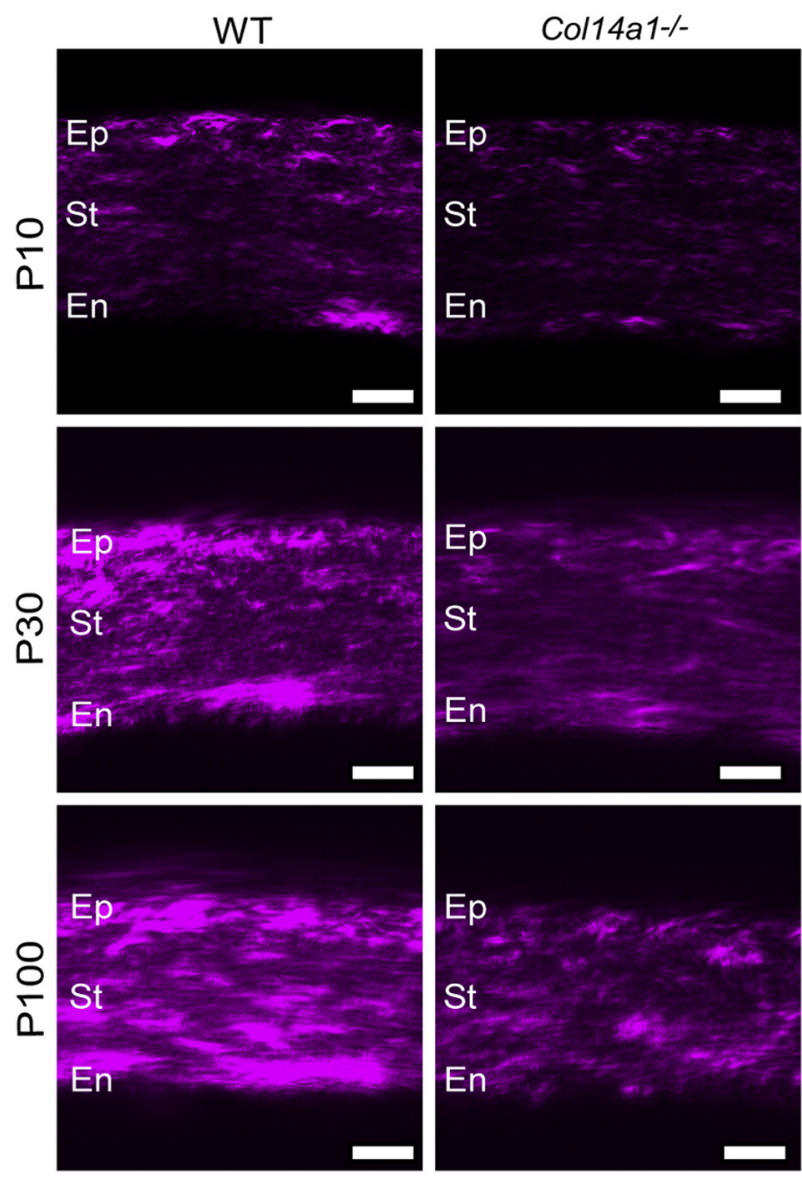

Figure 4 Second harmonic imaging of intact eye demonstrates impaired stromal compaction and suggests impaired fibrillogenesis in the absence of collagen XIV. Forward-scatter signal in wild-type C57BL/6 (WT) corneas at different ages (left column) compared with Col14a1 ${ }^{-/}$corneas (right column) shows stronger intensity in the WT corneas. $n=4$. Scale bars $=25 \mu \mathrm{m}$. En, endothelium; Ep, epithelium; P, postnatal day; St, stroma.

corneal laceration was performed from limbus to limbus using a custom-made diamond blade with a $70-\mu \mathrm{m}$ guarded depth (Mastel, Rapid City, SD). The stromal partial thickness laceration was performed in the horizontal axis of the eye. Corneal epithelial debridement was performed by deepithelializing the corneal surface with an AlgerBrush II bur (The Alger Company, Lago Vista, TX) over the central cornea until the epithelial layer was removed; at this point, the diamond bur was passed in three separate sweeps, with each causing visible abrasion. Immediately after the procedure, drops of moxifloxacin were applied to the ocular surface. ${ }^{17}$ Corneas from six different animals were used.

\section{Isolation and Culture of Corneal Fibroblasts}

After euthanasia, the eyes of P60 WT and Coll4al ${ }^{-1-}$ mice were copiously washed with betadine ophthalmic solution, and then incubated in Dulbecco's modified Eagle's medium containing $15 \mathrm{mg} / \mathrm{mL}$ Dispase II at $4^{\circ} \mathrm{C}$ for 18 hours. The entire corneal epithelium sheet loosened by this treatment was removed by vigorous shaking. Under a dissecting microscope, the corneal stroma was separated from the sclera at the corneoscleral limbus by pressing down the limbus with a 27-gauge needle. Isolated corneal stromas were incubated overnight at $37^{\circ} \mathrm{C}$ in Dulbecco's modified Eagle's medium containing $1.25 \mathrm{mg} / \mathrm{mL}$ collagenase A (Roche Applied Science) and $25 \mu \mathrm{g} / \mathrm{mL}$ gentamicin. A keratocyte-containing cell suspension was then seeded on T25 flasks (Thermo Fischer Scientific, Waltham, MA) in Dulbecco's modified Eagle's medium containing ITS (5 $\mu \mathrm{g} /$ $\mathrm{mL}$ insulin, $5 \mu \mathrm{g} / \mathrm{mL}$ transferrin, and $5 \mathrm{ng} / \mathrm{mL}$ sodium selenite), and $25 \mu \mathrm{g} / \mathrm{mL}$ gentamicin supplemented with $5 \%$ fetal bovine serum. When cells reached confluence, the same medium was switched to $1 \%$ fetal bovine serum and supplemented with $0.1 \mathrm{mmol} / \mathrm{L} \mathrm{L}$-ascorbic acid phosphate to enhance collagen synthesis and matrix formation for 10 days.

\section{Mass Spectrometry and Proteomics Analysis}

To analyze if collagen XIV influences the expression of major corneal stromal matrix components, WT and Coll $_{4 a 1^{-1-}}$ corneal fibroblasts were cultured, and protein extracts obtained from T25 flasks analyzed from a total of WT and Coll4a1 ${ }^{-1-}$ corneas were analyzed.

\section{Proteomic Analysis}

STRAP protein digestion: In brief, protein extracted from corneal fibroblasts expanded from WT and Coll4al ${ }^{-1-}$ stromas was lysed in $50 \mathrm{mmol} / \mathrm{L}$ triethylammonium bicarbonate buffer, $\mathrm{pH} 7.55$, containing 5\% SDS. To ensure no DNA remained to prevent the trap from clogging, the solution was then sheered thoroughly by probe sonication at 20\% amplitude, 5 seconds on and 5 seconds off, for 30 seconds. An S-Trap micro column (Protifi, Farmingdale, $\mathrm{NY}$ ) was placed in a $1.7-\mathrm{mL}$ tube to retain flow-through. The sample mixture was then added into the micro column $200 \mu \mathrm{L}$ at a time, followed by centrifugation of the micro column at $4000 \times g$ for 1 minute, removal of the flow-through, and repeating the process until the entire sample had passed through the S-Trap. The S-Trap micro

Table 1 SHG Forward-Scattered Signal Intensity during Stromal Development

\begin{tabular}{llll}
\hline WT & $567.219 \pm 33.9$ & $1393.8 \pm 196.9$ & $1526.1 \pm 151.6$ \\
Col14a1 & $406.4 \pm 61.8$ & $1069.4 \pm 121.8$ & $931.8 \pm 264$ \\
$P$ & 0.02 & 0.113 & 0.004 \\
\hline
\end{tabular}

Data are expressed as means \pm SD.

SHG, second harmonic generation; WT, wild-type C57BL/6. 

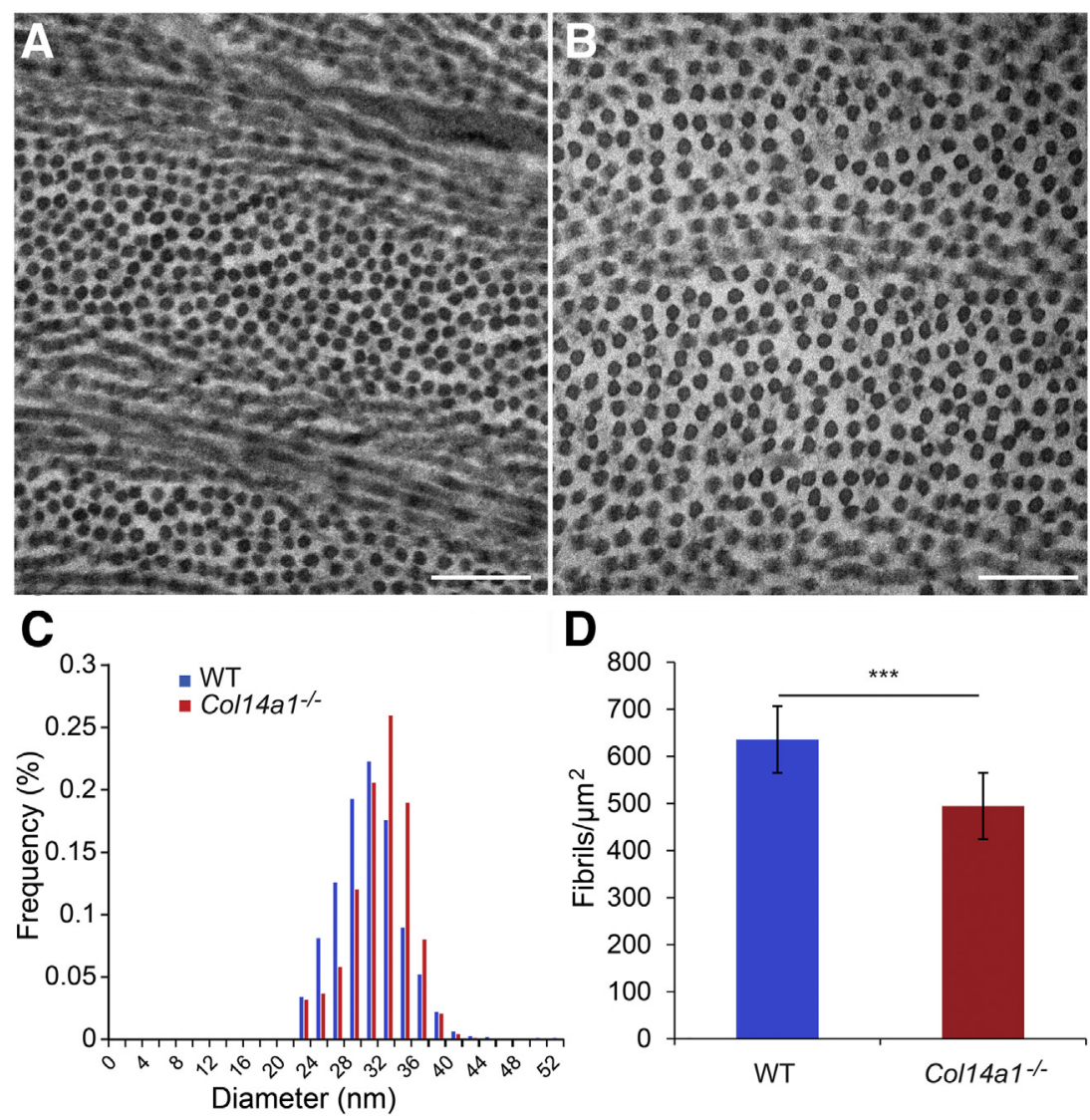

Figure 5 Collagen XIV is a regulator of fibril assembly during stromal maturation. A and B: Collagen fibril diameters are slightly larger in the absence of collagen XIV (B) than in the wild-type C57BL/6 (WT) corneal stroma (A). C: The mean fibril diameter in the C57BL/6 cornea is $30.7 \pm 3.8 \mathrm{~nm}$, and fibrils have a mean diameter of $32 \pm 3.5 \mathrm{~nm}$ in the absence of collagen XIV. D: The fibril density decreases significantly in Col14a1 ${ }^{-1-}$ stromas. $n=3$ (both groups). $* * * P<0.001$. Scale bars $=200 \mathrm{~nm}(\mathbf{A}$ and $\mathbf{B})$. column was then capped to limit evaporative loss without forming an airtight seal and incubated in a heat block for $37^{\circ} \mathrm{C}$ overnight. After digestion, peptides were eluted first with $40 \mu \mathrm{L}$ of $50 \mathrm{mmol} / \mathrm{L}$ triethylammonium bicarbonate and centrifugation at $4000 \times g$ for 1 minute. Peptides were characterized using a Thermo Q-exactive-HF mass spectrometer (Thermo Fisher Scientific) coupled to a Thermo Easy nLC 1200 (Thermo Fisher Scientific). The mass spectrometer was outfitted with a Thermo nanospray Flex source (Thermo Fisher Scientific) with the following parameters: spray voltage, 2.24; capillary temperature, $225^{\circ} \mathrm{C}$; and funnel RF level, 40. After protein identification, peptides for parallel reaction monitoring screening were chosen, the maximum time allowed for the instrument to acquire each specific ion was increased to 150 milliseconds, and the loop count was modified for the number of peptide masses chosen.

Table 2 Fibril Diameter in 30-Day-0ld Corneas

\begin{tabular}{lrcc}
\hline Fibril diameter, nm & WT & Col14a1 & $P$ value \\
\hline Mean & 30.7 & 32.0 & 0.026 \\
SD & 3.8 & 3.5 & \\
Median & 30.6 & 32.4 & \\
\hline
\end{tabular}

WT, wild-type C57BL/6.

\section{Statistical Analysis}

The $t$-test was used to draw statistical inferences when comparing the mean of continuous dependent variables. $P<$ 0.05 was considered statistically significant. Graph $\mathrm{Pad}$ Prism 9 software version 9.1.2 (GraphPad Software, San Diego, CA) was used for statistical calculations and heat map generation.

\section{Results}

Collagen XIV Is Highly Expressed in the Developing Corneal Stroma

The temporal pattern of Coll4al expression was assessed by studying Coll4al mRNA synthesis and collagen XIV

Table 3 Corneal Thickness Estimation Using Optical Coherence Tomography Imaging in Adult Corneas

\begin{tabular}{llll}
\hline Corneal thickness, $\mu \mathrm{m}$ & WT & Col14a1 & \\
\hline Mean & 104 & 82.2 & $P$ value \\
SD & 4.1 & 5.0 & $<0.0001$ \\
\hline
\end{tabular}

$n=8$ in each phenotype.

WT, wild-type C57BL/6. 
A Resistance to compression

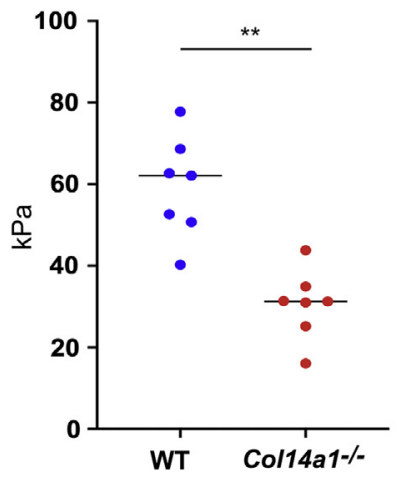

Young modulus ( $\mathrm{kPa})$

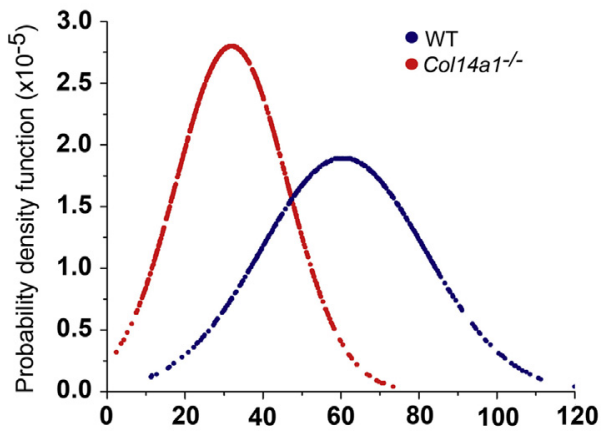

Figure 6 Collagen XIV influences corneal stiffness by increasing tissue resistance to compression. A: Wild-type C57BL/6 (WT) stromas were more resistant to compression than the Col14a1 ${ }^{-1-}$ stromas, with a significant decrease in Young's modulus and corneal stiffness without collagen XIV. B: The distribution of Young's modulus in Col14a1 ${ }^{-1-}$ corneas was narrower than that in WT corneas. Sixteen corneas were used in this experiment, eight WT and eight $\mathrm{Col}_{14 a 1^{-/-}}$. $* * P<0.01$. protein expression. The transcription of Coll4al in the cornea was analyzed from birth to adulthood in different stages of corneal development: immature (4 to 10 days old), preadult (30 days old), and adult (90 and 150 days old). The highest level of Coll4al mRNA expression occurred during immature development, at 4 to 10 days of age. In the preadult and adult stages, Coll4al mRNA expression decreased and a low level of Coll4al mRNA expression was present by 30 days of age and thereafter (Figure 1A). Protein immunoblot results were consistent with the mRNA analyses. Immature corneas at development demonstrated the highest expression of collagen XIV by WES simple Western analysis. Decreased collagen XIV expression was found at 30 days of age and thereafter (Figure 1B). In summary, Coll4al mRNA expression and collagen XIV protein content were higher during immature stages when stromal compaction occurs and decreased in the preadult stroma. This pattern of early expression and downregulation with maturation is consistent with an early function for collagen XIV as the cornea grows and attains function.

Collagen XIV Is Down-Regulated in the Corneal Stroma with Aging, but Its Expression Is Retained in the Adult Sclera

The spatial expression patterns of collagen XIV in the cornea and sclera were investigated using immunofluorescence microscopy. The findings demonstrated homogeneous collagen XIV throughout the cornea at early development, at 1 to 10 days of age. However, by $\geq 30$ days of age, collagen XIV was localized more in the anterior than posterior corneal stroma. At 90 days of age, there was no collagen XIV expression in the posterior stroma (Figure 2). In contrast, collagen XIV expression in the sclera, a stiffer tissue, was maintained at $\geq 90$ days of age (Figure 3). These findings suggest that collagen XIV expression is present in areas of higher tissue stiffness.
SHG Imaging Demonstrates Regulation of Fibrillogenesis and Stromal Compaction by Collagen XIV

Stromas with no collagen XIV were studied to assess the influence of collagen XIV on fibrillar collagen and hierarchical organization without tissue manipulation or chemical fixation. They were compared with WT stromas at different ages during development and in the adult cornea. Mean pixel intensity obtained from forward-scattered SHG signaling value was used as an approximation to fibril density and stromal compaction. A higher SHG forward signal intensity was detected in the WT compared with Coll4a1 ${ }^{-1-}$ stromas at all age groups studied. SHG forward-scattered signal intensity was decreased in the Coll4a1 $^{-1-}$ stroma (Figure 4). During development, WT stroma signaling intensity increased from 567.2 at P10 to 1393.8 at $\mathrm{P} 30$ and 1526.1 at $\mathrm{P} 100$, whereas $\mathrm{Coll4al}^{-/}$ stroma signaling intensity increased from 406.4 at P10 to 1069.4 at P30 and 931.8 at P100 (Table 1). These findings suggest that $\mathrm{Coll}_{4 a 1^{-1}}$ plays a significant role in collagen fibrillogenesis and regulation of collagen fibril microstructure.

\section{Collagen XIV Influences Fibril Diameter and Packing}

To analyze fibril assembly with an established and traditional technique, ultrastructural analyses were performed using TEM in Coll4al ${ }^{-1-}$ stromas with WT stromas as controls. Images were obtained and analyzed from the central cornea. ${ }^{19,20}$ At P30, fibrils in WT corneas were more uniform in shape and smaller than the fibrils in Coll4a1 ${ }^{-1-}$ corneas (Figure 5A). Coll4al ${ }^{-1-}$ corneas contained a higher frequency of larger diameter fibrils (Figure 5B). The presence of these slightly larger diameter fibrils increased the average diameter of the Coll4a1 ${ }^{-1-}$ collagen fibrils (Figure 5C). The average diameter of WT stromal collagen fibrils was $30.7 \pm 3.8 \mathrm{~nm}$, and the average diameter of Coll $_{4 I^{-1-}}$ collagen fibrils was $32.7 \pm 3.4 \mathrm{~nm}$. There was a 

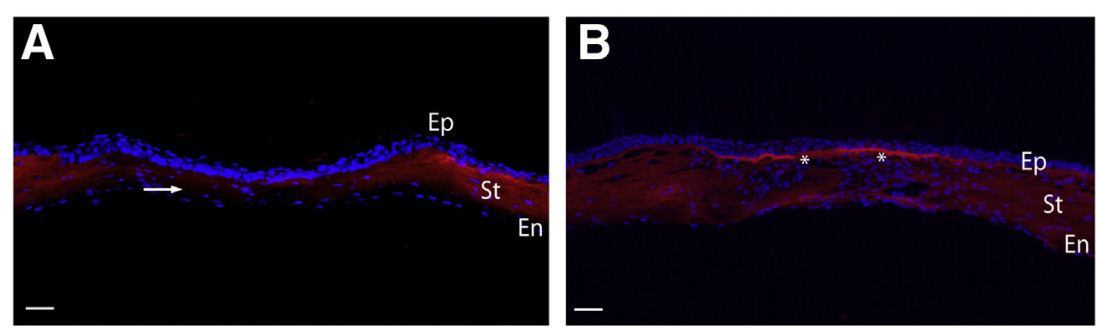

C

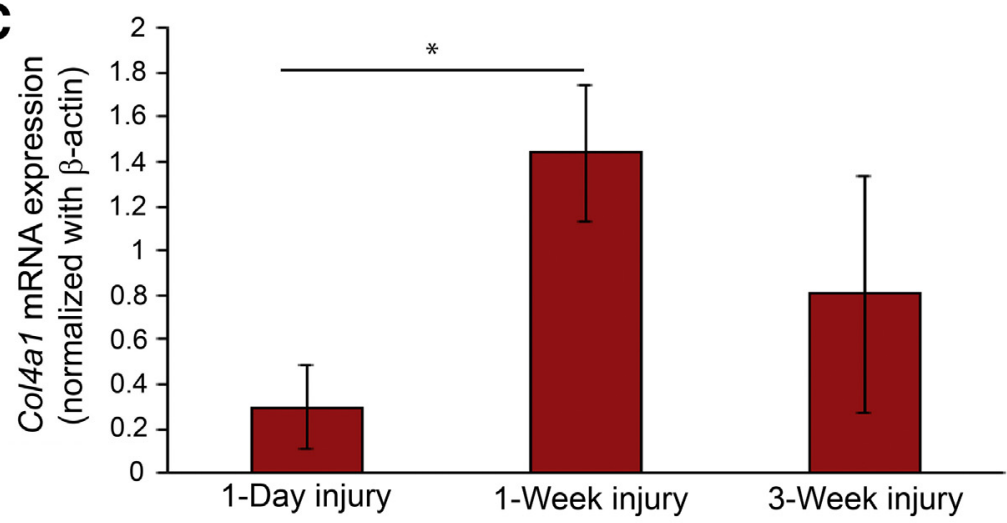

D

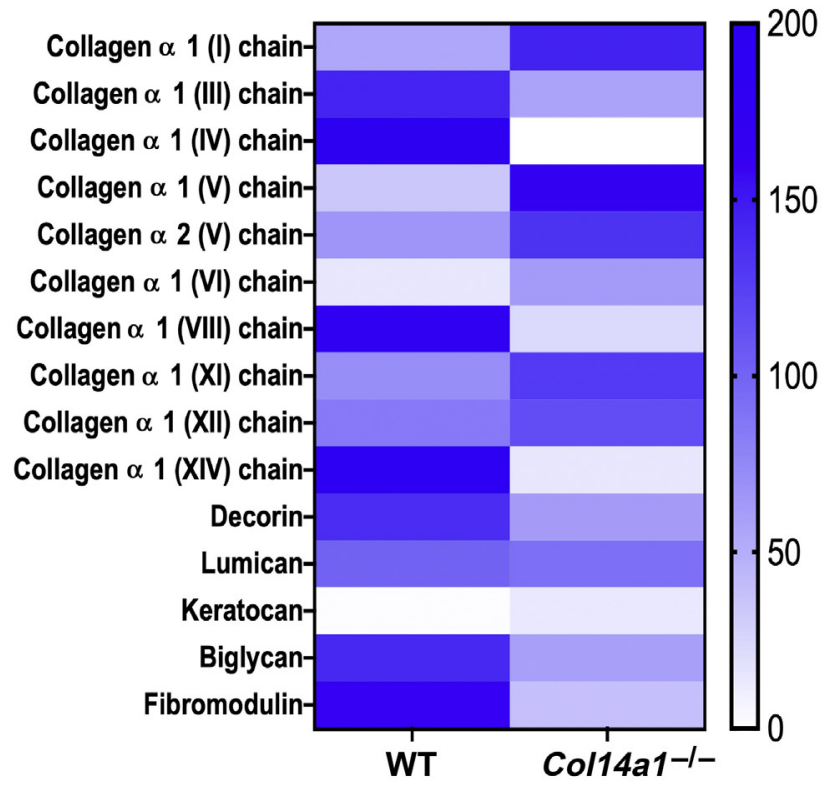

Figure 7 Collagen XIV expression is recapitulated following stromal injury. A and B: Images demonstrating loss of collagen XIV expression at 1 week, shown by arrow (A), followed by re-expression of collagen XIV, shown by asterisks, at 3 weeks (B). C: Real-time PCR shows up-regulation of Col14a1 mRNA expression during wound healing. D: Heat map demonstrates the effects of collagen XIV in influencing matrix composition by fibroblasts expanded in vitro; protein from 40 corneas was used in this experiment, and 20 wild-type C57BL/ 6 (WT) and 20 Col14a1 ${ }^{-/-}$ were used for in vitro expansion. $n=6(\mathbf{A}$ and $\mathbf{B})$; $n=3$ (C). ${ }^{*} P<0.05$. En, endothelium; Ep, epithelium; St, stroma. statistically significant difference in the average fibril diameters ( $t$-test, $P=0.026$ ) (Table 2$)$. Fibril density and the percentage area populated by fibrils in ROIs was quantified to indirectly assess fibril density and stromal compaction. There was a statistically significant difference in the fibril density and percentage area composed of fibrils in the ROI. The average fibril density for WT stromal collagen fibrils was $635 \pm 74.7$ fibrils $/ \mu \mathrm{m}^{2}$, whereas the average fibril density for Coll4al $^{-1-}$ stromal collagen fibrils was $494 \pm 48.6$ fibrils $/ \mu \mathrm{m}^{2}(P<0.001)$ (Figure 5D). The percentage area composed of fibrils in the WT stromal ROIs was $48 \% \pm 3.8 \%$, whereas the $\mathrm{Coll4al}^{-1-}$ stromal ROIs were $39.9 \% \pm 5.8 \%$ fibrils $(P<0.001)$. These findings suggest that collagen XIV plays a significant role in fibrillogenesis and stromal compaction, as shown by increased fibril density and compaction in the WT control.

\section{Collagen XIV Regulates Corneal Thickness in Adult Corneas}

To quantify if corneas had deposited more or less extracellular matrix during development, corneal thickness was measured in the adult animal, when the influence of an immature corneal endothelial monolayer is minimal. ${ }^{11} \mathrm{~A}$ statistically significant difference was found between Coll4al $^{-/-}$and WT adult corneal thickness. Mean corneal 
thickness in $\mathrm{Coll4al}^{-/}$was $82.2 \pm 5 \mu \mathrm{m}$; and in WT corneas, mean corneal thickness was $104.0 \pm 4.1 \mu \mathrm{m}$. There was a statistically significant difference as determined by unpaired $t$-test $(P<0.0001)$ (Table 3$)$. Taken together, these data demonstrate that collagen XIV is a regulator of stromal structure and hierarchical organization, affecting stromal thickness.

\section{Is Collagen XIV a Regulator of Stromal Stiffness and Mechanics?}

To determine whether the structural alterations noted by TEM and SHG microscopy in the absence of collagen XIV affect tissue mechanics and stromal function, corneal stiffness was tested in adult stromas to avoid effects of a dysfunctional immature endothelium in the absence of collagen XIV. ${ }^{11}$ Young's modulus was $59.2 \pm 12.4 \mathrm{kPa}$ for WT corneas and $30.5 \pm 8.5 \mathrm{kPa}$ for Coll $^{4} \mathrm{al}^{-1-}$ corneas (Figure 6A). The lack of collagen XIV resulted in corneas that were less resistant to compression by cantilever. The difference between the two groups was significant $(P=0.003, t$-test). The probability density function that describes the likelihood of occurrence for each value of Young's modulus also was analyzed. The distribution of Young's modulus in $\mathrm{CollHAl}^{-1-}$ corneas was narrower than that in WT corneas. It indicated that the stiffness of the Coll4al $^{-l-}$ corneas was more homogeneous than for the WT corneas (Figure 6B). These findings suggest that collagen XIV influences stromal resistance to compression and corneal mechanics.

\section{Is Collagen XIV Expression Recapitulated after Injury?}

To evaluate whether collagen XIV expression is recapitulated during wound healing, the stromal expression of collagen XIV was studied by fluorescence microscopy and mRNA Coll4al expression was quantified at weeks 1 and 3 after stromal injury using real-time PCR. Immunofluorescence microscopy showed loss of collagen XIV expression in the area of injury at 1 week (Figure 7A), but collagen XIV expression recapitulation was noted in the injured adult stroma at 3 weeks (Figure 7B). The transcription of Coll4al in the injured cornea increased significantly following injury (Figure 7C). Cultured corneal fibroblasts expanded in vitro, to mimic activated fibroblasts in vivo, showed significant modulation of multiple stromal extracellular matrix components by collagen XIV (Figure 7D).

\section{Discussion}

Intrinsic properties of cornea, including its transparency, thickness, and shape, depend on the interaction of collagen fibril synthesis and organization, a complex and highly orchestrated process that includes fibril assembly, fibril packing, and stromal compaction within the milieu of multiple extracellular matrix components. ${ }^{21-25}$ This study demonstrates that collagen XIV, a minor extracellular matrix protein in quantity, is expressed early and temporally during development. It may be an important regulator of stromal structure, and its expression during development likely affects key functional properties of the adult cornea.

The knowledge on the functions of FACITs, collagens XII and XIV, in the corneal stroma is limited; and their roles in establishing stromal structure and function are mostly unknown. A role in regulating fibrillogenesis during early development has been suspected. The temporal pattern of expression seen during avian corneal development suggests a regulatory role for collagen XIV in fetal and early postnatal corneal fibrillogenesis. ${ }^{6,15}$ A similar pattern has been reported during early development in tendon, but no effect in function in adult tendon was found. ${ }^{26}$ The current findings are consistent with a role for collagen XIV in regulation of fibrillogenesis during early development. This pattern of expression suggests that collagen XIV's main function is to regulate fibrillogenesis in periods of rapid tissue formation, like following stromal injury.

The regulatory roles of collagen XIV in keratocytes are unknown. FACITs influence multiple cell functions in the cornea. Corneal endothelial maturation is influenced by FACIT collagens, and it is plausible that endothelial immaturity might be partially responsible for increased interfibrillar spacing and increased corneal thickness noted in the null mouse model during early stages of development, ${ }^{11}$ However, the TEM fibril quantification findings and SHG signaling differences, together with alterations in corneal thickness and tissue mechanics noted at older ages, indicate that collagen XIV is a regulator of stromal structure and function per se. Collagen XII, a FACIT member of similar molecular structure, regulates cell function by multiple mechanisms. In osteoblasts, collagen XII deficiency induced cell disorganization by influencing cell polarity and disrupting cell-cell interactions. Decreased connexin 43 expression and impaired gap junction function were also noted. ${ }^{27}$ In the corneal stroma, collagen XII affects fibrillogenesis and keratocyte organization and regulates stromal stiffness. ${ }^{16}$ Collagen XII null stromas are characterized by decreased interfibrillar space and increased tissue stiffness. ${ }^{16}$ However, the effects of collagen XIV in stromal structure and function contrast with collagen XII. Collagen XIV null mice showed increased interfibrillar space and decreased tissue stiffness in this study.

Collagen XIV might be of translational importance in the pathogenesis of corneal ectasias based on three observations. First, ectatic diseases of the cornea are characterized by tissue weakening (decline in elastic modulus) that allows thinning, loss of tissue resistance, and corneal bulging. ${ }^{28}$ Collagen XIV is a prime candidate to study in the context of ectasia. The current findings using different techniques (eg, TEM, SHG, and atomic force microscopy) show that collagen XIV influences fibrillogenesis, stromal compaction, and therefore corneal stiffness. Second, the elastic modulus of mammalian cornea is three to four times higher 
in the anterior third of the stroma than in the rest of the cornea, implying that corneal resistance to deformation is relayed to this area. ${ }^{29,30}$ This is the region where collagen XIV expression persists the longest, whereas the first sign of ectasia is a change in the curvature of the posterior cornea. ${ }^{31}$ Interestingly, collagen XIV expression fades first in the posterior stroma. Third, collagen XIV expression also decreases significantly at the maturing stage of the murine stroma, a period when the clinical presentation of human keratoconus is florid. Interestingly, expression of collagen $\mathrm{XIV}$ in the stiffer and more rigid scleral tissue is maintained in the preadult and adult eye while being down-regulated in the cornea. One could hypothesize that decreased synthesis of collagen XIV or increased turnover of collagen XIV makes the corneal stroma more vulnerable to tissue weakening before natural cross-linking of the matrix hardens the corneal stroma.

Finally, collagen XIV may have not only structural but also signaling functions by binding other matrix components or by influencing cell functions directly by integrin binding. It is known that collagen XIV can bind decorin, ${ }^{32}$ tenascin $\mathrm{X},{ }^{33}$ and heparin. ${ }^{34}$ The finding that collagen XIV expression is recapitulated in the adult during wound healing and the significant proteome changes noted in the absence of collagen XIV suggest that this FACIT is needed to re-establish wound strength and function. Futures studies are needed to better understand the role it plays during stromal wound healing.

The importance of other types of collagens in stromal function is not well understood either. Most studies have focused on the role of collagen $\mathrm{V}$ in stromal development. ${ }^{25}$ Not much is known about the role(s) of collagens VI, XI, XII, and XIV in different functions of the cornea, including stromal shape and thickness, and their role in preventing neovascularization or injury repair. The current knowledge on their role on ectatic disorders of the cornea or in the process of wound healing is minimal. Further studies are needed to explore the role of fibrillar and FACIT collagens normally expressed in the corneal stroma.

In summary, collagen XIV expression is restricted to the early stages of normal stromal development and appears to influence fibrillogenesis and stromal compaction, with accompanying changes in corneal function in the adult stroma.

\section{References}

1. Mienaltowski MJ, Birk DE: Structure, physiology, and biochemistry of collagens. Adv Exp Med Biol 2014, 802:5-29

2. Gordon MK, Castagnola P, Dublet B, Linsenmayer TF, Van der Rest M, Mayne R, Olsen BR: Cloning of a cDNA for a new member of the class of fibril-associated collagens with interrupted triple helices. Eur J Biochem 1991, 201:333-338

3. Shaw LM, Olsen BR: FACIT collagens: diverse molecular bridges in extracellular matrices. Trends Biochem Sci 1991, 16:191-194

4. Agarwal P, Zwolanek D, Keene DR, Schulz JN, Blumbach K, Heinegard D, Zaucke F, Paulsson M, Krieg T, Koch M, Eckes B:
Collagen XII and XIV, new partners of cartilage oligomeric matrix protein in the skin extracellular matrix suprastructure. J Biol Chem 2012, 287:22549-22559

5. Walchli C, Trueb J, Kessler B, Winterhalter KH, Trueb B: Complete primary structure of chicken collagen XIV. Eur J Biochem 1993, 212:483-490

6. Young BB, Zhang G, Koch M, Birk DE: The roles of types XII and XIV collagen in fibrillogenesis and matrix assembly in the developing cornea. J Cell Biochem 2002, 87:208-220

7. Watt SL, Lunstrum GP, McDonough AM, Keene DR, Burgeson RE, Morris NP: Characterization of collagen types XII and XIV from fetal bovine cartilage. J Biol Chem 1992, 267:20093-20099

8. Lethias C, Descollonges Y, Garrone R, van der Rest M: Expression of type XIV collagen during the differentiation of fetal bovine skin: immunolabeling with monoclonal antibody is prominent in morphogenetic areas. J Invest Dermatol 1993, 101:92-99

9. Ansorge HL, Meng X, Zhang G, Veit G, Sun M, Klement JF, Beason DP, Soslowsky LJ, Koch M, Birk DE: Type XIV collagen regulates fibrillogenesis: premature collagen fibril growth and tissue dysfunction in null mice. J Biol Chem 2009, 284:8427-8438

10. Tao G, Levay AK, Peacock JD, Huk DJ, Both SN, Purcell NH, Pinto JR, Galantowicz ML, Koch M, Lucchesi PA, Birk DE, Lincoln J: Collagen XIV is important for growth and structural integrity of the myocardium. J Mol Cell Cardiol 2012, 53:626-638

11. Hemmavanh C, Koch M, Birk DE, Espana EM: Abnormal corneal endothelial maturation in collagen XII and XIV null mice. Invest Ophthalmol Vis Sci 2013, 54:3297-3308

12. Brown JC, Golbik R, Mann K, Timpl R: Structure and stability of the triple-helical domains of human collagen XIV. Matrix Biol 1994, 14: 287-295

13. Berthod F, Germain L, Guignard R, Lethias C, Garrone R, Damour O, van der Rest M, Auger FA: Differential expression of collagens XII and XIV in human skin and in reconstructed skin. J Invest Dermatol 1997, 108:737-742

14. Niyibizi C, Visconti CS, Kavalkovich K, Woo SL: Collagens in an adult bovine medial collateral ligament: immunofluorescence localization by confocal microscopy reveals that type XIV collagen predominates at the ligament-bone junction. Matrix Biol 1995, 14: $743-751$

15. Gordon MK, Foley JW, Lisenmayer TF, Fitch JM: Temporal expression of types XII and XIV collagen mRNA and protein during avian corneal development. Dev Dyn 1996, 206:49-58

16. Sun M, Zafrullah N, Devaux F, Hemmavanh C, Adams S, Ziebarth NM, Koch M, Birk DE, Espana EM: Collagen XII is a regulator of corneal stroma structure and function. Invest Ophthalmol Vis Sci 2020, 61:61

17. Cogswell D, Sun M, Greenberg E, Margo CE, Espana EM: Creation and grading of experimental corneal scars in mice models. Ocul Surf 2020, 19:53-62

18. Espana EM, Kawakita T, Di Pascuale MA, Li W, Yeh LK, Parel JM, Liu CY, Tseng SC: The heterogeneous murine corneal stromal cell populations in vitro. Invest Ophthalmol Vis Sci 2005, 46:4528-4535

19. Sun M, Chen S, Adams SM, Florer JB, Liu H, Kao WW, Wenstrup RJ, Birk DE: Collagen V is a dominant regulator of collagen fibrillogenesis: dysfunctional regulation of structure and function in a corneal-stroma-specific Col5a1-null mouse model. J Cell Sci 2011, 124:4096-4105

20. Chen S, Young MF, Chakravarti S, Birk DE: Interclass small leucinerich repeat proteoglycan interactions regulate collagen fibrillogenesis and corneal stromal assembly. Matrix Biol 2014, 35:103-111

21. Maurice DM: The structure and transparency of the cornea. J Physiol 1957, 136:263-286

22. Linsenmayer TF, Fitch JM, Gordon MK, Cai CX, Igoe F, Marchant JK, Birk DE: Development and roles of collagenous matrices in the embryonic avian cornea. Prog Retin Eye Res 1998, 17: $231-265$

23. Hassell JR, Birk DE: The molecular basis of corneal transparency. Exp Eye Res 2010, 91:326-335 
24. Chen S, Mienaltowski MJ, Birk DE: Regulation of corneal stroma extracellular matrix assembly. Exp Eye Res 2015, 133:69-80

25. Espana EM, Birk DE: Composition, structure and function of the corneal stroma. Exp Eye Res 2020, 198:108137

26. Young BB, Gordon MK, Birk DE: Expression of type XIV collagen in developing chicken tendons: association with assembly and growth of collagen fibrils. Dev Dyn 2000, 217:430-439

27. Izu Y, Sun M, Zwolanek D, Veit G, Williams V, Cha B, Jepsen KJ, Koch M, Birk DE: Type XII collagen regulates osteoblast polarity and communication during bone formation. J Cell Biol 2011, 193: $1115-1130$

28. Blackburn BJ, Jenkins MW, Rollins AM, Dupps WJ: A review of structural and biomechanical changes in the cornea in aging, disease, and photochemical crosslinking. Front Bioeng Biotechnol 2019, 7:66

29. Dias JM, Ziebarth NM: Anterior and posterior corneal stroma elasticity assessed using nanoindentation. Exp Eye Res 2013, 115: $41-46$
30. Winkler M, Chai D, Kriling S, Nien CJ, Brown DJ, Jester B, Juhasz T, Jester JV: Nonlinear optical macroscopic assessment of 3-D corneal collagen organization and axial biomechanics. Invest Ophthalmol Vis Sci 2011, 52:8818-8827

31. Belin MW, Asota IM, Ambrosio R Jr, Khachikian SS: What's in a name: keratoconus, pellucid marginal degeneration, and related thinning disorders. Am J Ophthalmol 2011, 152:157-162.e1

32. Font B, Aubert-Foucher E, Goldschmidt D, Eichenberger D, van der Rest M: Binding of collagen XIV with the dermatan sulfate side chain of decorin. J Biol Chem 1993, 268:25015-25018

33. Lethias C, Carisey A, Comte J, Cluzel C, Exposito JY: A model of tenascin-X integration within the collagenous network. FEBS Lett 2006, 580:6281-6285

34. Giry-Lozinguez C, Aubert-Foucher E, Penin F, Deleage G, Dublet B, van der Rest M: Identification and characterization of a heparin binding site within the NC1 domain of chicken collagen XIV. Matrix Biol 1998, 17:145-149 